\title{
Comunicación de la responsabilidad social corporativa (RSC): análisis de las campañas más premiadas en 2018
}

Communicating corporate social responsibility (CSR): an analysis of the most award-winning campaigns of 2018

\author{
Elisenda Estanyol
}

Cómo citar este artículo:

Estanyol, Elisenda (2020). "Comunicación de la responsabilidad social corporativa (RSC): Análisis de las campañas más premiadas en 2018". Profesional de la información, v. 29, n. 3, e290334.

https://doi.org/10.3145/epi.2020.may.34

Artículo recibido el 16-03-2020 Aceptación definitiva: 01-04-2020

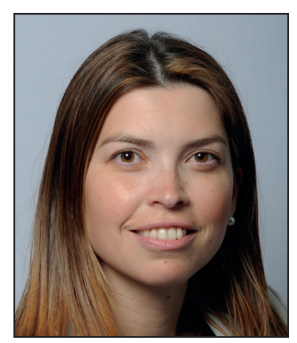

\author{
Elisenda Estanyol \\ http://orcid.org/0000-0003-3986-0377 \\ Universitat Oberta de Catalunya \\ Estudios de Ciencias de la Información y de \\ la Comunicación \\ Avda. Tibidabo, 39-43. \\ 08035 Barcelona, España \\ eestanyol@uoc.edu
}

\section{Resumen}

La responsabilidad social corporativa (RSC) tiene un impacto en la reputación de las empresas y de las instituciones, lo que convierte su gestión en crucial también desde el punto de vista comunicativo. Cada vez más, los stakeholders -no únicamente los consumidores-, piden a las organizaciones que se pronuncien sobre los principales problemas que afectan a la sociedad y que actúen con causa, por lo que la comunicación de la RSC está en constante evolución. El propósito de esta investigación es examinar prácticas exitosas de comunicación de RSC a partir del estudio de las campañas más premiadas en 2018. Se ha aplicado como metodología de investigación el análisis de contenido cualitativo. Concretamente, se han analizado los certámenes PR Daily Corporate Social Responsibility Awards, Cannes Lions PR Lions e IPRA Golden World Awards, centrándose el estudio en las acciones y programas premiados en las categorías de RSC, siendo éstas un total de 29. Las categorías analizadas han sido: sujeto promotor, sector, zona geográfica, sujeto ejecutor, tema, objetivo, público/s y técnicas, tácticas y canales utilizados. El análisis de los resultados ha permitido detectar prácticas exitosas como: la alineación de las acciones de RSC con los objetivos de desarrollo sostenible (ODS) propuestos por la ONU (siendo los más recurrentes la igualdad de género y el empoderamiento de la mujer, la reducción de las desigualdades y la protección del medio ambiente); la vinculación de los temas de RSC con la misión y propósito de las organizaciones (especialmente en empresas del sector tecnológico que buscan la promoción de vocaciones científico-tecnológicas entre las jóvenes); la involucración de los empleados en las acciones de RSC (lo que implica una concepción más integral de su gestión); y, por último, el uso de canales cada vez más bidireccionales para favorecer el diálogo entre las organizaciones y sus públicos de interés.

\section{Palabras clave}

Responsabilidad social corporativa; RSC; Relaciones públicas; RRPP; Premios; Comunicación; Reputación; Objetivos de desarrollo sostenible; ODS.

\footnotetext{
Abstract

Corporate social responsibility (CSR) has an impact on the reputation of companies and institutions, so its management is also crucial from a communicative perspective. Stakeholders -not just consumers- demand that companies take a position on the main problems that affect society and act conscientiously, resulting in a constant evolution of CSR communication. The purpose of this research is to examine successful CSR communication practices based on a study of the most award-winning campaigns of 2018. Qualitative content analysis is applied as the research methodology. Specifically, the PR Daily Corporate Social Responsibility Awards, Cannes Lions PR Lions, and IPRA Golden World Awards
} 
are analyzed, focusing on a total of 29 actions and programs awarded in the CSR categories. The categories analyzed are client, sector, geographic scope, implementer, topic, objective, public(s), and techniques, tactics, and channels used. The analysis of the results reveals successful practices such as the alignment of CSR actions with the UN sustainable development goals (SDGs) (especially gender equality and women's empowerment, reduction of inequalities, and environmental protection), the linking of CSR issues with the mission and purpose of organizations (especially by technology companies that seek to promote scientific-technological careers among young women), the involvement of employees in the implementation of CSR actions (which shows a more comprehensive conception of CSR management), and finally, the use of bidirectional channels to favor dialogue between organizations and their stakeholders.

\section{Keywords}

Corporate social responsibility; CSR; Public relations; PR; Awards; Communication; Reputation; Sustainable development goals; SDG.

\section{Introducción}

La responsabilidad social corporativa (RSC) se considera un activo de valor que debe gestionarse de forma estratégica y transversal, y que repercute en la reputación de las organizaciones (Morsing; Schultz; Nielsen, 2008; Virakul; Koonmee; McLean, 2009; Kim, 2019).

\section{Definida como}

"una forma de dirigir las empresas basada en la gestión de impactos que su actividad genera sobre sus clientes, trabajadores, accionistas, comunidades locales, medio ambiente y sobre la sociedad en general" (ORSC, 2019), enfatiza la necesidad de ir más allá del cumplimiento de la ley y se diferencia del concepto de filantropía (Castillo-Esparcia, 2009) por tener un fin estratégico vinculado a la misión de la organización.

Los cambios acelerados propiciados por las nuevas tecnologías y la globalización son algunos de los factores principales que han impulsado la RSC. Otros son:

- interés de los consumidores por las empresas y los productos éticos y presión organizada por parte de ONGs, sindicatos y asociaciones de consumidores (Canyelles-Pastó, 2016);

- visibilidad de las buenas (y malas) praxis con la irrupción de las redes sociales;

- "creciente sensibilidad de la sociedad por el desarrollo empresarial y el deterioro medioambiental" (Barrio-Fraile, 2019, p. 15);

- "impulso legislativo ejercido por organismos públicos y privados" (Milian-Dueñas, 2015, p. 3);

- preocupación de las empresas por obtener legitimación social (Herrera-Morillas; Castillo-Díaz; Pérez-Pulido, 2014).

Los Objetivos de desarrollo sostenible (ODS) marcados en la Agenda 2030, promovidos por las Naciones Unidas y adoptados por sus estados miembros en 2015, han ayudado también a situar la RSC en el núcleo de la gestión de muchas empresas.

Desde la perspectiva de las relaciones públicas, la RSC ha sido siempre un área de especialización clave en tanto que afecta a la reputación, un intangible que se ve afectado por el comportamiento de la organización y por la forma en la que gestiona sus relaciones con los stakeholders o grupos de interés (Xifra, 2014). Sin embargo, a pesar del importante rol que juega la comunicación, Kim apunta que

"desde la literatura se ha prestado poca atención a los aspectos comunicativos de la RSC" (Kim, 2019, p. 1.143).

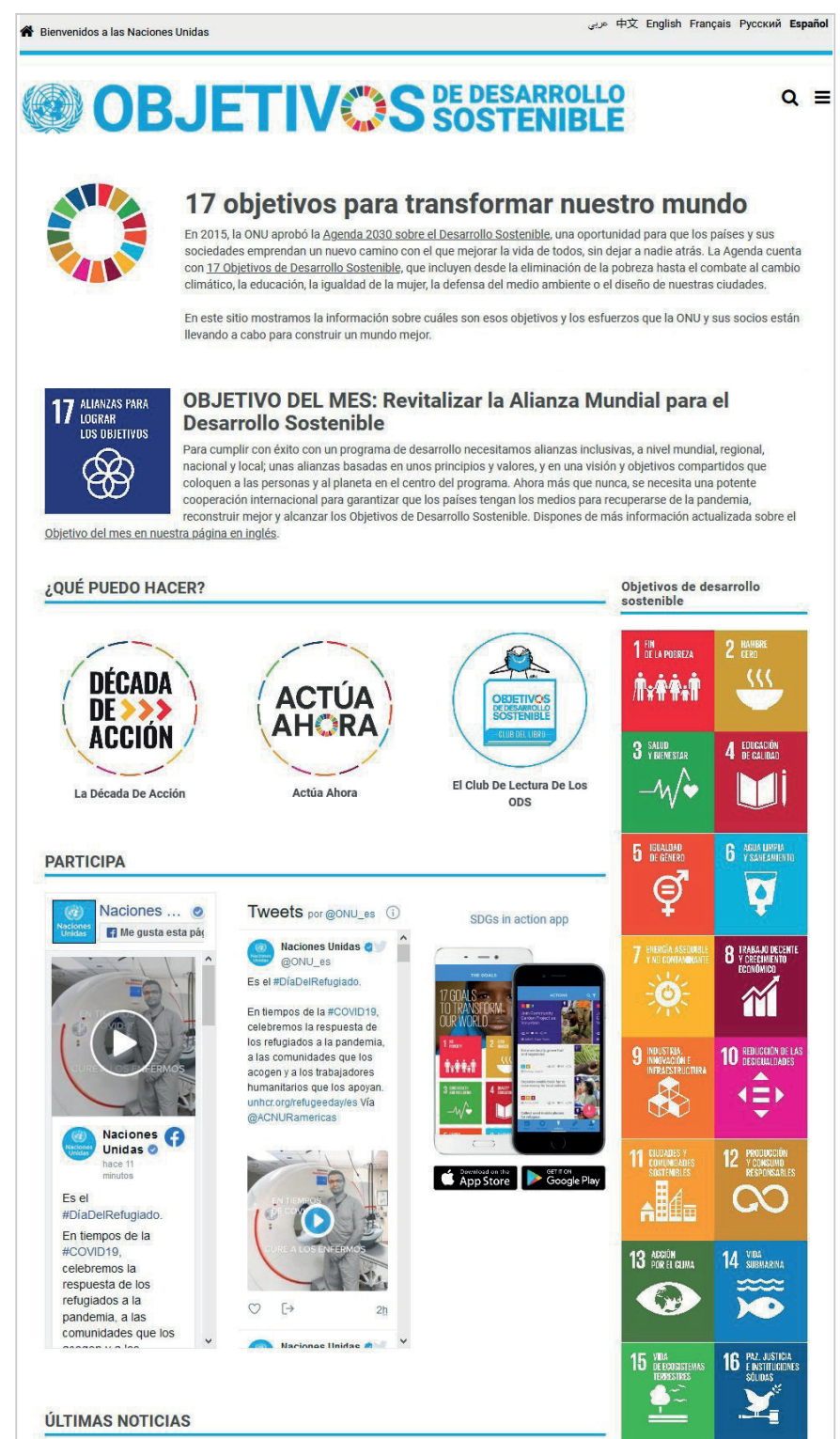

https://www.un.org/sustainabledevelopment/es 
Desde la práctica profesional, las organizaciones tienen dificultades para aplicar el concepto abstracto de RSC a su realidad (Rahbek-Pedersen, 2006). Si bien la bibliografía especializada está llena de estudios de caso sobre cómo se ha llevado a cabo en corporaciones y entidades de todo tipo, como señalan Rungsuka y Sirathanakulb (2013) es necesario identificar prácticas contemporáneas exitosas, y analizar cómo se comunican con los públicos de interés (Orozco-Toro; Ferré-Pavia, 2013). Se detecta también una escasez de investigaciones sobre los premios en RSC que vayan más allá de focalizarse en una única campaña (Ahmad, 2016).

Esta investigación persigue cubrir parte de este vacío, examinando algunas de las mejores acciones comunicativas de RSC a partir de un análisis de las campañas más premiadas en certámenes internacionales en 2018. El estudio tiene como objetivo identificar a qué públicos se dirigen, en qué países se han implementado, cuáles son los sujetos ejecutores de las mismas y sobre todo, cuáles son los temas más recurrentes, para así descubrir si están alineados con los ODS.

\section{Marco teórico}

\subsection{La RSC en el marco de la teoría de los grupos de interés}

La teoría de los grupos de interés (o stakeholders) se ha convertido en el paradigma dominante en el estudio de la RSC (Morsing; Schultz, 2006; Crane; Glozer, 2016; Albasu; Nyameh, 2017).

\section{Freeman definió el término stakeholder como}

"cualquier grupo o individuo que puede afectar o ser afectado por el logro de los objetivos de la empresa" (Freeman, 1984, p. 24).

Se pasaba así de una visión de las empresas centradas en los consumidores y accionistas, a considerar todo el abanico de públicos con quienes las organizaciones interactúan y ante quienes son responsables (empleados, ONGs, proveedores, sociedad, medios de comunicación, etc.). Los stakeholders tienen el potencial de ayudar o dañar a la empresa (Gibson, 2000), por lo que no deben contemplarse solamente como impactados por la actividad empresarial sino como interlocutores y agentes activos en su implantación.

La teoría de los stakeholders fue adoptada por la disciplina de las Relaciones Públicas, sobre todo en la bibliografía anglosajona (Grunig; Hunt, 1984; Ferguson, 1984; Clark, 2000; Franketal, 2001; Bartlett; Tywoniak; Hatcher, 2007; Kim; Reber, 2008), concibiéndola como el establecimiento de relaciones mutuamente beneficiosas entre las organizaciones y todos sus públicos. Xifra (2014) recuerda que las organizaciones están bajo el foco de la opinión pública, y que deben rendir cuentas no sólo ante sus inversores sino también ante la ciudadanía, quien fiscaliza si cumplen con sus obligaciones sociales. En definitiva, para que las organizaciones puedan influir en la agenda de RSC, su comportamiento al respecto debe comunicarse eficazmente a los públicos (Moreno; Capriotti, 2006).

Desde la bibliografía se ha subrayado la importancia de los públicos internos para la gestión exitosa de la RSC (Morsing; Schultz; Nielsen, 2008; Du; Battacharya; Sen, 2010; Coombs; Holladay, 2012); aunque para autores como Crane y Glozer (2016) todavía falta por impulsar el papel de los empleados como actores clave en su ejecución.

En consecuencia, la RSC no puede limitarse a la comunicación y a las acciones de Relaciones Públicas si éstas no están entrelazadas con un comportamiento ético por parte de la organización y sus directivos (McWilliams; Siegel, 2001), concibiéndose como parte esencial de la empresa y gestionándose de forma integral y estratégica a largo plazo (Toro, 2006; Aceituno-Aceituno et al., 2013).

\section{2. $\mathrm{RSC}$ y reputación}

Entre todos los intangibles corporativos, la reputación, definida como la imagen mental de una organización que evoluciona a través del tiempo como resultado de su comportamiento y comunicación efectiva (Fombrun, 1996), se ha convertido en el más relevante para contribuir al éxito de las organizaciones (Smith, 2003; Truñó-Gual; Rialp-Criado, 2008; Pérez, 2015).

Para autores como Morsing, Schultz y Nielsen (2008) la RSC tiene una implicación directa en la reputación de las organizaciones. Virakul, Koonmee y McLean (2009) apuntan que la mejora de la reputación es uno de los motivos esgrimidos por las empresas para ser más socialmente responsables, aunque no puede convertirse en una acción de maquillaje o de

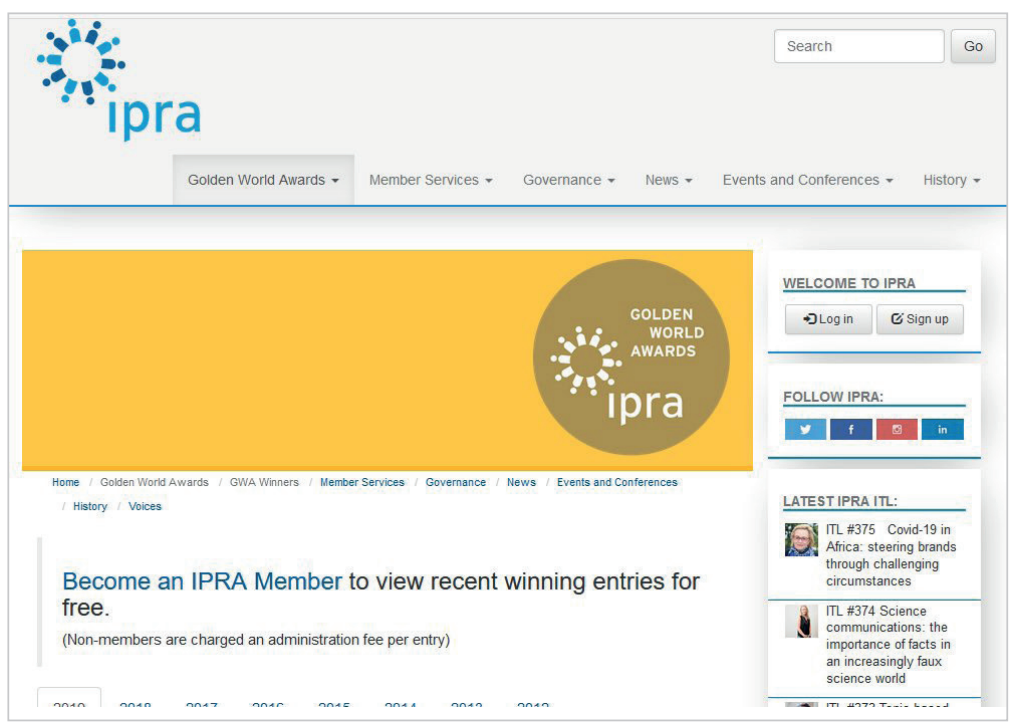

https://www.ipra.org/golden-world-awards/winners 
greenwashing. La RSC repercutirá favorablemente en la imagen de la organización siempre que su comunicación sea transparente y verificable (Pérez, 2015). Hay sin embargo otros autores que consideran que la RSC es un factor necesario, pero no suficiente de la reputación (Kim; Park, 2011; Melo; Garrido-Morgado, 2012), esgrimiendo que ésta depende también de factores como el sector en el que opere cada organización y el grado de notoriedad del que previamente ya disponga.

Conseguir credibilidad entre los stakeholders es un importante antecedente de la reputación corporativa (Fombrun, 1996). Para ello, se recomienda que las relaciones y la comunicación entre organizaciones y stakeholders se basen en la confianza (Du; Bhattacharya; Sen, 2010; Coombs; Holladay, 2012) y que las acciones de RSC estén vinculadas con el core business de la empresa (Simmons; Becker-Olsen, 2006), con la misión y la visión de la organización (Toro, 2006)

\subsection{Importancia de la comunicación de la RSC}

La comunicación regular con los stakeholders es un elemento considerado por la mayoría de autores como esencial en el diseño y la implementación de la RSC (Du; Bhattacharya; Sen, 2010; Eberle; Berens; Li, 2013). Aún así, tal y como subrayan Crane y Glozer (2016), la bibliografía sobre RSC ha enfatizado más la comunicación hacia los públicos que con ellos.

El concepto de diálogo, clave en la teoría de las Relaciones Públicas -subrayado en el modelo simétrico bidireccional formulado por Grunig y Hunt (1984) y estudiado en el contexto digital por Kent y Taylor, (1998) y Kent (2013)-, lo es también en la teoría de los stakeholders de la RSC, que enfatiza la necesidad de conseguir una involucración proactiva por parte de los públicos, para conseguir así una comunicación basada en el diálogo constante (Barlett; Devin, 2011).

Para evitar la comunicación unidireccional (de la organización a sus públicos) y perseguir el entendimiento mutuo, los autores animan a usar canales como la intranet y especialmente las redes sociales (Orozco; Ferré, 2013; Illia et al., 2015). Así mismo, la independencia de la fuente a través de la cual se comunica la RSC tiene también un impacto en la credibilidad de la comunicación, siendo mayor si el medio es externo, en vez de uno propio y controlado por la propia organización (Eberle; Berens; Li, 2013).

A continuación se presenta un análisis de las campañas más premiadas en RSC para identificar si estos postulados y recomendaciones que emanan de la bibliografía se están aplicando en el ámbito profesional a la hora de comunicar eficazmente las acciones de RSC.

\section{Metodología}

El objetivo principal de este trabajo es identificar prácticas exitosas de comunicación de la RSC para descubrir aspectos comunes. Los objetivos específicos son:

O1. Determinar cuáles son los temas más frecuentes de las campañas de comunicación de RSC más premiadas y analizar si éstos están en línea con los objetivos de desarrollo sostenible (ODS).

O2. Conocer a qué públicos se dirigen de forma más mayoritaria, y qué técnicas, tácticas y canales utilizan en mayor medida.

Los certámenes que se han estudiado han sido:

- IPRA Golden World Awards, organizados por la Asociación Internacional de Relaciones Públicas; https://www.ipra.org/golden-world-awards/winners

- Cannes Lions PR, promovidos por el Festival Internacional de Creatividad de Cannes; http://www.canneslionsarchive.com/Home/PublicHome

- PR Daily Corporate Social Responsibility Awards, organizados por la revista digital PR Daily. https://www.prdaily.com/awards/corporate-social-responsibility-awards/2018/winners

La investigación se ha centrado en las acciones y programas premiados en 2018 en las categorías de RSC. Se han escogido estos premios por ser internacionales, del ámbito de la comunicación y de las relaciones públicas, y por contar con categorías específicas de RSC. 
La metodología de investigación aplicada ha sido el análisis de contenido, entendido como

"una técnica de investigación destinada a formular, a partir de ciertos datos, inferencias reproducibles y válidas que puedan aplicarse a su contexto" (Krippendorff, 1990, p. 28).

El enfoque adoptado ha sido cualitativo, realizando un análisis descriptivo e interpretativo. La muestra la conforman 29 acciones de RSC: la totalidad de campañas premiadas en categorías de RSC en estos certámenes.

Se han establecido categorías y subcategorías de análisis, partiendo de los principales elementos que componen las campa-

ñas de comunicación, según han sido definidos por autores referentes de la disciplina de las relaciones públicas (Cutlip; Center; Broom, 2006; Wilcox y Cameron, 2012; Smith, 2017). Se han identificado los sujetos que conforman el proceso comunicativo:

- promotor: describiéndose también el sector económico;

- ejecutor: clasificándolo en dos subcategorías: departamento de comunicación integrado en la organización -in houseo agencia externa);

- receptor: identificando el público específico al que se ha dirigido cada campaña.

Partiendo también de la clasificación realizada por estos autores, se han identificado las técnicas, tácticas y canales de comunicación más frecuentemente adoptados.

Para la codificación de los temas de cada campaña, se ha analizado la propia descripción de las acciones realizada por las entidades que las han promovido, estudiando su finalidad manifiesta e identificando las palabras clave. La información se ha extraído del palmarés de los premios y de las propias páginas web de los sujetes promotores y los sujetos ejecutores de la campaña. Por último, para determinar si estos temas están alineados con los ODS, se han codificado teniendo en cuenta el número asociado a cada uno de los diecisiete objetivos establecidos por la ONU.

Las unidades de registro se han codificado de forma sistemática partiendo de las categorías y subcategorías definidas, transformando la información en códigos alfanuméricos. Posteriormente, se ha realizado un análisis de frecuencia para determinar las características y prácticas más comunes (en el Anexo 1 se detalla el sistema de categorías y subcategorías).

\section{Resultados}

\subsection{Sujeto promotor, sujeto ejecu- tor y área geográfica}

Los sujetos promotores de las campañas de RSC más premiadas, es decir aquellas empresas o instituciones que las han impulsado y financiado, son de diversa índole y pertenecen a sectores económicos muy variados (ver Anexo 1). Esta heterogeneidad pone de relieve que la RSC es una práctica que no se enmarca en organizaciones de un sector concreto.

Las acciones de RSC han sido ideadas y ejecutadas por profesionales de la comunicación y de las relaciones públicas, ya sean integrados en los departamentos de comunicación de las empresas (in-house) (aproximadamente 2/3) o en agencias de

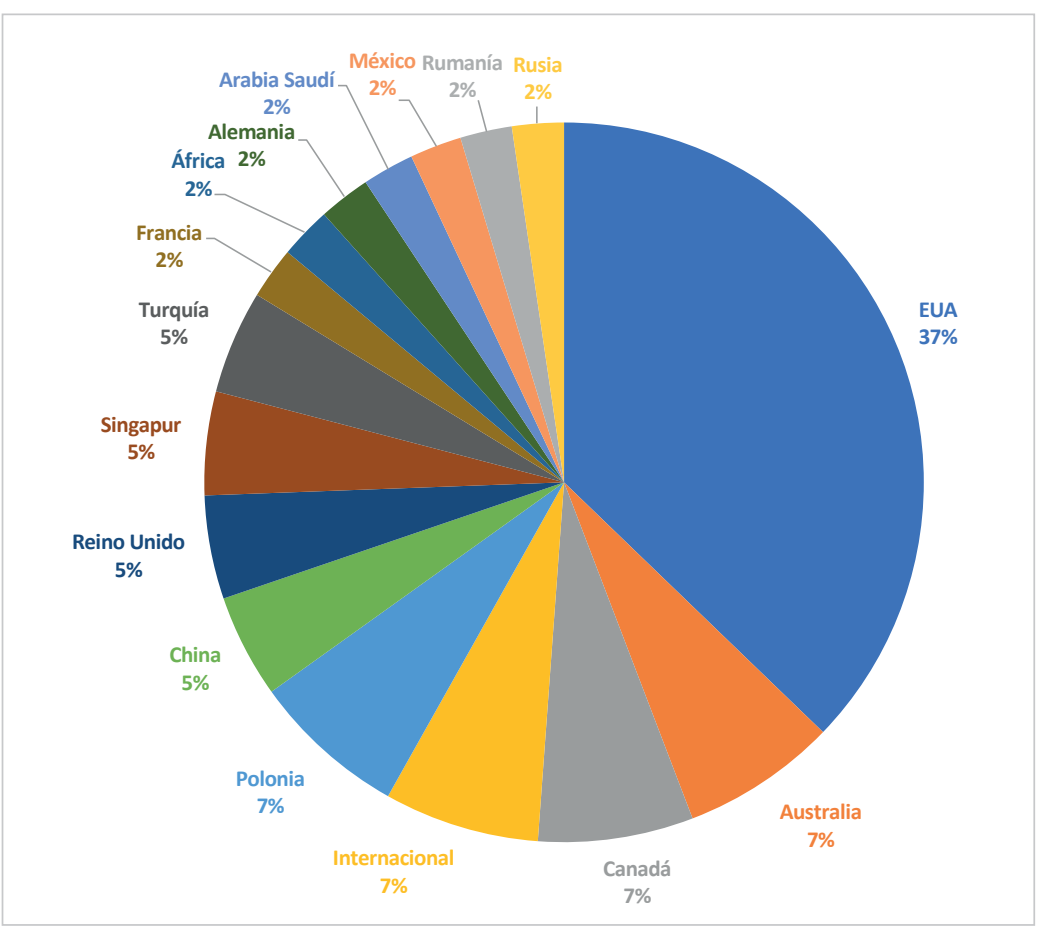

Gráfico 1. Área geográfica de implantación de las campañas de RSC 
comunicación y Relaciones Públicas (entre las que destacan Burson Cohn \& Wolfe, Lobby PR y Yulu Public Relations) (1/3), constatando que se trata de un área de especialización que se ha implantado en el sector de la comunicación corporativa, pero con un mayor predominio de ejecución interna, por su fuerte componente-como veremos- de relación con los empleados.

En referencia a las áreas geográficas, se observa un claro dominio en la implantación de los programas de RSC en países anglosajones (Estados Unidos, Australia y Canadá), seguidos de Polonia y Turquía. Hay muy pocas campañas premiadas de Latinoamérica, a excepción de México (gráfico 1). La mayor presencia de acciones de RSC en países anglosajones puede deberse a una apuesta más decidida de sus empresas, aunque podría también ser porque sus profesionales tengan más costumbre de presentarse a certámenes internacionales. Por otro lado, la proporción de campañas internacionales es reducida, lo que muestra que en la RSC es importante el arraigo social de las organizaciones con sus comunidades más cercanas.

\subsection{Temas}

El tema más frecuente ha sido la promoción de las vocaciones científico-tecnológicas, de ingeniería y matemáticas (en su acrónimo STEM en inglés) [TB]. Presente en 6 de las 29 campañas que conforman la muestra, estas acciones han sido lideradas sobre todo por empresas del sector tecnológico-industrial y dirigidas al alumnado de secundaria, en muchos casos específicamente a estudiantes del sexo femenino. Se trata de campañas que cuentan con la colaboración de centros formativos, pero también de los propios empleados de las compañías tecnológicas, quienes participan impartiendo talleres o charlas. Estas acciones van en la línea de revertir el hecho de que actualmente las mujeres sólo representen el 30\% de los empleos del sector tecnológico (OECD, 2019). También a la llamada de éste y otros organismos económicos internacionales para cubrir con graduados STEM los millones de empleos que desaparecerán en 2030 como consecuencia de la automatización, la digitalización y la inteligencia artificial (Nübler, 2017; World Economic Forum, 2018; OECD, 2019).

El segundo tema más común ha sido la promoción de la igualdad de género y el empoderamiento de la mujer [TA] (4 de 29), seguido por la protección del medio ambiente [TC], el fomento de la igualdad de oportunidades, la salud de las personas, la reducción del hambre, y la ayuda a los damnificados por catástrofes naturales (centradas, sobre todo en ayudas después de los huracanes Harvey e Irma). El resto de las campañas premiadas tienen finalidades múltiples, como la lucha contra el acoso escolar, el racismo, la promoción de una alimentación más saludable y la reducción de los accidentes de tráfico. Todo ello muestra un mayor foco en la responsabilidad social y de compromiso con las comunidades cercanas, que en la ambiental. También se demuestra que en la mayoría de campañas, los temas de RSC están estrechamente vinculados con la misión de las compañías que las promueven y que tienen informada en sus webs (gráfico 2).

\subsection{Objetivos de desarrollo sostenible (ODS)}

De los 17 objetivos de desarrollo sostenible (ODS) propuestos por la ONU (2019) y adoptados por sus estados miembros en la denominada Agenda 2030, observamos cómo en las campañas de RSC más premiadas en 2018 el más frecuente fue el que persigue la "Igualdad de género y el empoderamiento de la mujer" (ODS 5), concretamente en 7 de las campañas que conforman la muestra (gráfico 3). El impulso del movimiento feminista en 2017 y la notoriedad del movimiento \#MeToo, pueden haber favorecido este auge. Algunas de las campañas que se enmarcan en este ODS son:

- The flip impulsada por McDonald's [C4];

- Equal dictionary de Arçelik implantada en Turquía [I3];

- \#SheDrives, que Nissan diseñó para Arabia Saudí para incentivar la conducción entre las mujeres [C2].

Los siguientes ODS más comunes entre las campañas más premiadas son también sociales. De hecho, la lucha contra el cambio climático y la protección del medio ambiente (enmarcado en el ODS 13) han centrado sólo dos campañas:

- Save our species de Lacoste [C3], que tenía como objetivo proteger a especies en peligro de extinción;

- Life of a can, impulsada por Novelis [P18], para promover el reciclaje. 


\subsection{Públicos}

Si nos fijamos específicamente en los públicos a los que de forma mayoritaria se han dirigido las campañas premiadas, vemos que aparte de las dirigidas a la concienciación de la población en general [AS]-, como públicos específicos se encuentran los estudiantes de secundaria (particularmente del sexo femenino) [AA] (en 8 de las 29 acciones) y los niños menores de 12 años [AB] (en 4 de 29). Las mujeres (sobre todo las empleadas) [AD] y los damnificados por catástrofes naturales [AC] son otros de los públicos más impactados. Les siguen las poblaciones en riesgo de exclusión social y los enfermos (gráfico 4).

En las campañas premiadas destaca la dimensión interna de la RSC. En casi una tercera parte, los empleados son actores principales, actuando como profesores invitados en escuelas, contribuyendo económicamente con ONGs o implantando una política de voluntariado corporativo más amplia y que entraña que los trabajadores dediquen parte de su tiempo en proyectos para la mejora de las comunidades locales. Los trabajadores de las empresas se convierten así en stakeholders principales en términos de RSC, no sólo como destinatarios sino también como embajadores y partícipes de su implementación. En el caso de Berkshire Bank [P17] por ejemplo, los responsables de comunicación detectaron que había aumentado hasta un $80 \%$ el deseo de sus empleados de continuar trabajando en la entidad, se había incrementado el sentimiento de pertenencia, la cohesión entre los equipos y la mejora en la relación con los directores.

\subsection{Técnicas, tácticas y canales}

Aparte de las relaciones con los medios de comunicación para generar publicity [SM] -técnica de relaciones públicas todavía ampliamente utilizada y exitosa en las campañas de comunicación de RSC analizadas-, se observa una apuesta por la organización de eventos [SE], muchos de ellos enmarcados en programas de comunicación interna.

A nivel de tácticas, destacan el storytelling y el video content, así como la colaboración con celebrities. Por ejemplo, en la acción Be a buddy not a bully [I1] impulsada por la cadena Turner Broadcasting Cartoon Network en África con el objetivo de reducir el acoso escolar, se escogió como protagonista a un atleta paralímpico que había sido víctima de bulling. También se han identificado acciones de gamificación -por ejemplo, en la [12]-, entendida como

"la integración de dinámicas de juego en entornos no lúdicos" (Deterding et al., 2011).

En lo que respecta a los canales de comunicación más utilizados, sobresalen los propios de las empresas (webs, blogs, intranets y newsletters), pero también la creación de aplicaciones específicas para dispositivos móviles y las redes sociales (básicamente Facebook, Twittter e Instagram). En aquellas campañas dirigidas al público joven, predomina el uso de YouTube. Todo ello, muestra una tendencia hacia el uso de canales menos unidireccionales y que permiten una interlocución más directa con los públicos.

\section{Discusión y conclusiones}

La RSC, como elemento de valor estratégico, debe gestionarse de forma integral dentro de las organizaciones, siendo la comunicación una parte importante de esta gestión coordinada (Du; Bhattacharya; Sen, 2010). El valor clave de la comunicación en la RSC se plasma en su práctica, tanto por parte de departamentos de comunicación integrados en las 
organizaciones, como por consultoras externas (sujetos ejecutores). Así lo corrobora la existencia de categorías específicas de RSC en premios internacionales del sector como los PR Daily, los Cannes Lions y los IPRA Golden Awards. El análisis de las campañas más premiadas en la edición de 2018 de estos certámenes permite detectar prácticas exitosas implantadas en varios países.

Los temas de las campañas de RSC analizadas están intrínsecamente ligados en su mayoría al propósito y la misión de las organizaciones que las promueven, lo que según autores como Simmons y Becker-Olsen (2006), ayuda a que éstas sean percibidas de forma más creíble por los stakeholders y así contribuir a la mejora de la reputación, un intangible clave no sólo a nivel de relaciones públicas, sino empresarial. En las acciones más premiadas analizadas, se comprueba que esta recomendación se aplica sobre todo en dos sectores empresariales: el tecnológico y el automovilístico. En el primero, con acciones que promueven las vocaciones entre jóvenes para estudiar carreras STEM, y en el segundo, con iniciativas que buscan reducir la mortalidad en accidentes de tráfico.

Se detecta también que los objetivos de las campañas de RSC analizadas están alineados con los ODS definidos por la ONU, siendo el ODS 5 que persigue la igualdad de género y el empoderamiento de la mujer, el más recurrente en las campañas premiadas, seguido del ODS 10, que busca reducir las desigualdades entre países y dentro de ellos y el ODS 13 que promueve adoptar medidas contra el cambio climático. Ello demuestra que las organizaciones están atentas a las demandas y movimientos sociales, como el movimiento feminista y el que denuncia la emergencia climática, pero que también apuestan por aquellas acciones de RSC que las preparan para los desafíos económicos del futuro.

En casi la mitad de las campañas más premiadas, los empleados han sido actores principales de la ejecución de las acciones de RSC, lo que pone de manifiesto que muchas organizaciones han dejado de entender la RSC como una práctica orientada principalmente a los públicos externos (accionistas y clientes, principalmente), para ser cada vez más conscientes de la importancia estratégica de involucrar a los públicos internos para dotarla de mayor legitimidad, como promueven autores como Morsing, Schultz y Nielsen (2008), Coombs y Holladay (2012) y Crane y Glozer (2016).

A nivel de técnicas de relaciones públicas, la organización de eventos se alza como una de las más aplicadas, aunque también está generalizada la relación con los medios de comunicación. La colaboración con celebrities y el video content con voluntad de viralización difundido a través de las redes sociales, se alzan también como una de las tácticas más destacadas a la hora de relacionarse sobre todo con los públicos más jóvenes. En este sentido, el uso de canales menos unidireccionales, como los blogs y las redes sociales, con ejemplos claros en las campañas \#SheDrives y \#GiveAToast, afianza la búsqueda de una interacción más directa con los públicos de interés, tal y como se subraya desde la teoría de los stakeholders (Freeman, 1984; Gibson, 2000; Morsing; Schultz, 2006; Crane; Glozer, 2016).

Se puede concluir que en la comunicación de la RSC de las campañas más premiadas, se aplican algunas de las recomendaciones postuladas desde la bibliografía, aunque quedan otras aún pendientes de implantar, sobre todo las dirigidas a establecer un diálogo real entre organización y públicos de interés (Kent, 2013). Del mismo modo, los canales utilizados en las campañas han sido básicamente los propios de las organizaciones, lejos de aplicarse la recomendación de autores como Eberle, Berens y Li (2013) de utilizar medios externos para conseguir mayor credibilidad gracias a la independencia atribuida a la fuente.

Las prácticas exitosas identificadas: alineación de las acciones de RSC con los ODS, vinculación de los temas de RSC con la misión y propósito de las organizaciones, arraigo con las comunidades más cercanas, involucración de los empleados y uso de canales bidireccionales, pueden tener también implicaciones prácticas para el sector profesional.

\section{Referencias}

Aceituno-Aceituno, Pedro; Cea-Moure, Ramiro; Casado-Sánchez, José-Luís; Ruiz-de-Azcárate-Varela, Carmen (2013). "La comunicación como factor clave en la implantación de la responsabilidad social corporativa: el caso de Crédit Agricole España". El profesional de la información, v. 22, n. 4, pp. 326-332.

https://doi.org/10.3145/epi.2013.jul.08

Ahmad, Zeti-Azreen (2016). "Communicating CSR in the digital age: An exploratory study of a CSR award winning company in Malaysia". Journal of education and social sciences, v. 4, pp. 252-257.

https://www.jesoc.com/wp-content/uploads/2016/08/SS-96.pdf

Albasu, Joseph; Nyameh, Jerome (2017). "Relevance of stakeholders theory, organizational identity theory and social exchange theory to corporate social responsibility and employees performance in the commercial banks in Nigeria". International journal of business, economics and management, v. 4, n. 5, pp. 95-105.

https://doi.org/10.18488/journal.62.2017.45.95.105

Barrio-Fraile, Estrella (2019). Responsabilidad social corporativa. De la noción a la gestión. Barcelona: Editorial UOC. ISBN: 9788491804093

Bartlett, Jennifer L.; Devin, Bree (2011). "Management, communication and corporate social responsibility". In: Ihlen, $\emptyset$ yvind; Bartlett, Jennifer L.; May, Steve (eds.). The handbook of communication and corporate social responsibility. Sussex: John Wiley \& Sons, pp. 47-66. ISBN: 9781444336344

https://doi.org/10.1002/9781118083246.ch3 
Bartlett, Jennifer; Tywoniak, Stephane; Hatcher, Caroline (2007). "Public relations professional practice and the institutionalisation of CSR". Journal of communication management, v. 11, n. 4, pp. 281-299.

https://doi.org/10.1108/13632540710843904

Cannes Lions. Cannes Lions PR Lions Winners 2018.

http://www.canneslionsarchive.com/Home/PublicHome

Canyelles-Pastó, Josep-Maria (2016). El nuevo ambiente de responsabilidad social del negocio. Barcelona: Editorial UOC. http://openaccess.uoc.edu/webapps/o2/bitstream/10609/49144/2/El\%20nuevo\%20ambiente\%20de\%20 Responsabilidad\%20Social\%20del\%20negocio.pdf

Castillo-Esparcia, Antonio (2009). Relaciones públicas. Teoría e historia. Barcelona: Editorial UOC. ISBN: 9788497888172

Clark, Cynthia E. (2000). "Differences between public relations and corporate social responsibility: An analysis". Public relations review, v. 26, n. 3, pp. 363-380.

https://doi.org/10.1016/s0363-8111(00)00053-9

Coombs, W. Timothy; Holladay, Sherry J. (2012). Managing corporate social responsibility: A communication approach. Malden, MA: Wiley-Blackwell. ISBN: 9781444336290

Crane, Andrew; Glozer, Sarah (2016). "Researching corporate social responsibility communication: themes, opportunities and challenges". Journal of management studies, v. 53, n. 7, pp. 1223-1252.

https://doi.org/10.1111/joms.12196

Cutlip, Scott M.; Center, Allen H.; Broom, Glen M. (2006). Effective public relations. San Diego: Pearson. ISBN: 9780 132450775

Deterding, Sebastian; Dixon, Dan; Khaled, Rilla; Nacke, Lennart (2011). "From game design elements to gamefulness: defining 'gamification'". In: Proceedings of the $15^{\text {th }}$ International academic mindtrek conference, pp. 9-15.

https://doi.org/10.1145/2181037.2181040

Du, Shuili; Bhattacharya, C. B.; Sen, Sankar (2010). "Maximizing business returns to corporate social responsibility (CSR): the role of CSR communication". International journal of management reviews, v. 12, n. 1, pp. 8-19.

https://doi.org/10.1111/j.1468-2370.2009.00276.x

Eberle, David; Berens, Guido; Li, Ting (2013). "The impact of interactive corporate social responsibility communication on corporate reputation". Journal of business ethics, n. 118, pp. 731-746.

https://doi.org/10.1007/s10551-013-1957-y

Ferguson, Mary-Ann (2018). "Building theory in public relations: interorganizational relationships as a public relations paradigm". Journal of public relations research, v. 30, n. 4, pp. 164-178.

https://doi.org/10.1080/1062726X.2018.1514810

Fombrun, Charles J. (1996). Reputation: realizing value from the corporate image. Boston: Harvard Business School Press. ISBN: 9780875846330

Franketal, Peter (2001). “Corporate social responsibility - a PR invention?”. Corporate communications: An international journal, v. 6, n. 1, pp. 18-23.

https://doi.org/10.1108/13563280110381170

Freeman, R. Edward (1984). Strategic management. A stakeholder approach. London: Pitman. ISBN: 9780521151740

Gibson, Kevin (2000). "The moral basis of stakeholder theory". Journal of business ethics, v. 26, pp. 245-257. https://doi.org/10.1023/A:1006110106408

Grunig, James E.; Hunt, Todd T. (1984). Managing public relations. New York: Holt, Rinehart \& Winston. ISBN: 9780 030583377

Herrera-Morillas, José-Luis; Castillo-Díaz, Ana; Pérez-Pulido, Margarita (2014). “Responsabilidad social y sostenibilidad en las bibliotecas universitarias españolas". El profesional de la información, v. 23, n. 2, pp. 134-143.

https://doi.org/10.3145/epi.2014.mar.05

Illia, Laura; Romenti, Stefania; Rodríguez-Cánovas, Belén; Murtarelli, Grazia; Carroll, Craig E. (2017). “Exploring corporations' dialogue about CSR in the digital era". Journal of business ethics, n. 146, v. 1, pp. 39-58.

https://doi.org/10.1007/s10551-015-2924-6

IPRA. IPRA Golden World Awards Winners 2018.

https://www.ipra.org/golden-world-awards/winners

Kent, Michael L. (2013). "Using social media dialogically: public relations role in reviving democracy". Public relations review, v. 39, n. 4, pp. 337-345.

https://doi.org/10.1016/j.pubrev.2013.07.024 
Kent, Michael L.; Taylor, Maureen (1998). "Building dialogic relationships through the World Wide Web”. Public relations review, n. 24, v. 3, pp. 321-334.

https://doi.org/10.1016/S0363-8111(99)80143-X

Kim, Soo-Yeon; Park, Hyojung (2011). “Corporate social responsibility as an organizational attractiveness for prospective public relations practitioners". Journal of business ethics, v. 103, n. 4, pp. 639-653.

https://doi.org/10.1007/s10551-011-0886-x

Kim, Soo-Yeon; Reber, Bryan (2008). "Public relations' place in corporate social responsibility: practitioners define their role". Public relations review, v. 34, n. 4, pp. 337-342.

https://doi.org/10.1016/j.pubrev.2008.07.003

Kim, Sora (2019). "The process model of corporate social responsibility (CSR) communication: CSR communication and its relationship with consumers' CSR knowledge, trust, and corporate reputation perception". Journal of business ethics, v. 154 , n. 4, pp. 1143-1159.

https://doi.org/10.1007/s10551-017-3433-6

Krippendorff, Klaus (1990). Metodología de análisis de contenido. Teoría y práctica. Barcelona: Paidós Comunicación. ISBN: 8475096271

McWilliams, Abagail; Siegel, Donald (2001). "Corporate social responsibility: a theory of the firm perspective". Academy of management review, v. 26, n. 1, pp. 117-127.

http://www.jstor.org/stable/259398

Melo, Tiago; Garrido-Morgado, Álvaro (2012). "Corporate reputation: a combination of social responsibility and industry". Corporate social responsibility and environmental management, v. 19, n. 1, pp. 11-31.

https://doi.org/10.1002/csr.260

Milian-Dueñas, Laura (2015). Responsabilidad social corporativa: origen y evolución del concepto de RSC en el entorno empresarial europeo y español. Madrid: Universidad Pontificia Comillas.

Moreno, Ángeles; Capriotti, Paul (2006). “La comunicación de las empresas españolas en sus webs corporativas: análisis de la información de responsabilidad social, ciudadanía corporativa y desarrollo sostenible”. Zer, n. 21, pp. 49-64.

https://www.ehu.eus/ojs/index.php/Zer/article/view/3718

Morsing, Mette; Schultz, Majken (2006). “Corporate social responsibility communication: stakeholder information, response and involvement strategies". Business ethics: A European review, v. 15, n. 4, pp. 323-338.

https://doi.org/10.1111/j.1467-8608.2006.00460.x

Morsing, Mette; Schultz, Majken; Nielsen, Kasper-Ulf (2008). "The 'Catch 22' of communicating CSR: Findings from a Danish study". Journal of marketing communications, v. 14, n. 2, pp. 97-111.

https://doi.org/10.1080/13527260701856608

Nübler, Irmgard (2017). “Las nuevas tecnologías y las dinámicas de creación de empleo”. Organización Internacional del Trabajo, 3 mayo.

https://www.ilo.org/global/about-thilo/newsroom/news/WCMS_552360/lang--es/index.htm

OECD (2019). OECD Future of education and skills 2030. Organisation for Economic Co-operation and Development.

http://www.oecd.org/education/2030

ONU (2019). Objetivos de desarrollo sostenible (ODS).

https://www.un.org/sustainabledevelopment/es/gender-equality

Orozco-Toro, Jaime-Alberto; Ferré-Pavia, Carme (2013). "La comunicación estratégica de la responsabilidad social corporativa". Razón y palabra, n. 83, pp. 242-258.

http://www.razonypalabra.org.mx/N/N83/V83/20_OrozcoFerre_V83.pdf

ORSC (2019). Qué es RSC. Observatorio de Responsabilidad Social Corporativa.

https://observatoriorsc.org/la-rsc-que-es

Pérez, Andrea (2015). “Corporate reputation and CSR reporting to stakeholders: Gaps in the literature and future lines of research". Corporate communications: An international journal, v. 20, n. 1, pp. 11-29.

https://doi.org/10.1108/CCIJ-01-2014-0003

PR Daily (2019). PR daily corporate social responsibility awards 2018 winners.

https://www.prdaily.com/awards/corporate-social-responsibility-awards/2018/winners

Rahbek-Pedersen, Esben (2006). "Making corporate social responsibility (CSR) operable: How companies translate stakeholder dialogue into practice". Business and society review, v. 111, n. 2, pp. 137-163.

https://doi.org/10.1111/j.1467-8594.2006.00265.x 
Rungsuka, Anong; Sirathanakulb, Kumron (2013). "CSR activities of multinational corporations in Thailand". Academic journal of management sciences, v. 2, n. 1, pp. 36-43

Simmons, Carolyn J.; Becker-Olsen, Karen L. (2006). "Achieving marketing objectives through social sponsorships". Journal of marketing, n. 70, pp. 154-169.

https://doi.org/10.1509/jmkg.70.4.154

Smith, N. Craig (2003). “Corporate social responsibility: Whether or how?”. California management review, v. 45, n. 4, pp. 52-76.

https://doi.org/10.2307/41166188

Smith, Ronald D. (2017). Strategic planning for public relations. Routledge. ISBN: 9780415994224

Toro, Daniela (2006). “El enfoque estratégico de la responsabilidad social corporativa: revisión de la literatura académica". Intangible capital, v. 2, n. 14, pp. 338-358.

https://upcommons.upc.edu/handle/2099/2942

Truñó-Gual, Jordi; Rialp-Criado, Josep (2008). "La responsabilidad social corporativa: Gestión empresarial de un activo intangible". Revista de contabilidad y dirección, v. 7, pp. 163-184.

https://accid.org/wp-content/uploads/2018/10/rcd7_castella_163-1.pdf

Virakul, Busaya; Koonmee, Kalayanee; McLean, Gary N. (2009). "CSR activities in award-winning Thai companies". Social responsibility journal, v. 5, n. 2, pp. 178-199.

https://doi.org/10.1108/17471110910964478

Wilcox, Dennis; Cameron, Glen T. (2012). Public relations: strategies and tactics. Washington: Pearson. ISBN: 9780 205960644

World Economic Forum (2018). The global competitiveness report 2018.

https://www.weforum.org/reports/the-global-competitveness-report-2018

Xifra, Jordi (2014). Manual de relaciones públicas e institucionales. Madrid: Tecnos. ISBN: 9788430952670

Anexo 1. Tabla de análisis de las acciones de RSC premiadas en 2018

\begin{tabular}{|c|c|c|c|c|c|c|c|c|c|c|}
\hline Premio & Acción & $\begin{array}{l}\text { Sujeto } \\
\text { promotor }\end{array}$ & $\begin{array}{c}\text { Sector económico } \\
\text { del cliente }\end{array}$ & $\begin{array}{l}\text { Sujeto } \\
\text { ejecutor }\end{array}$ & $\begin{array}{c}\text { Área } \\
\text { geográfica }\end{array}$ & $\begin{array}{l}\text { Público } \\
\text { principal }\end{array}$ & $\begin{array}{l}\text { Tema } \\
\text { RSC }\end{array}$ & ODS & $\begin{array}{l}\text { Técnicas } \\
\text { y tácticas }\end{array}$ & Canales \\
\hline [11] & $\begin{array}{l}\text { Be a buddy not a } \\
\text { bully }\end{array}$ & $\begin{array}{l}\text { Cartoon } \\
\text { Network }\end{array}$ & $\begin{array}{l}\text { Canal de } \\
\text { televisión }\end{array}$ & $\begin{array}{l}\text { [a] Bur- } \\
\text { son-Mars- } \\
\text { teller }\end{array}$ & $\begin{array}{l}\text { África (todos } \\
\text { países } \\
\text { africanos) }\end{array}$ & {$[A B]$} & {$[\mathrm{TE}]$} & [ODS10] & {$[\mathrm{SC}],[\mathrm{SV}]$} & {$[\mathrm{CB}]$} \\
\hline$[12]$ & Bring the kindness & $\begin{array}{l}\text { Amway } \\
\text { charity } \\
\text { foundation }\end{array}$ & Marketing & $\begin{array}{l}\text { [a] Mint } \\
\text { (BBDO } \\
\text { Rusia) }\end{array}$ & Rusia & {$[\mathrm{AB}]$} & [TO $]$ & [ODS1] & {$[\mathrm{SE}]$} & {$[\mathrm{CB}]$} \\
\hline$[13]$ & Equal dictionary & Arçelik & Electrodomésticos & [a] Lobby PR & Turquía & {$[\mathrm{AS}]$} & {$[\mathrm{TA}]$} & [ODS5] & {$[\mathrm{SG}],[\mathrm{TV}]$} & {$[\mathrm{CB}]$} \\
\hline$[14]$ & $\begin{array}{l}\text { Turkey's engineer } \\
\text { girls }\end{array}$ & $\begin{array}{l}\text { Limak } \\
\text { Foundation }\end{array}$ & Construcción & [in] & Turquía & {$[\mathrm{AA}]$} & {$[\mathrm{TB}]$} & [ODS5] & {$[\mathrm{SS}]$} & {$[\mathrm{CU}]$} \\
\hline$[\mathrm{C} 1]$ & $\begin{array}{l}\text { The most German } \\
\text { Supermarket }\end{array}$ & $\begin{array}{l}\text { Edeka } \\
\text { Zentrale AG } \\
\& \text { CO. KG }\end{array}$ & Supermercados & $\begin{array}{l}\text { [a] Jug Von } \\
\text { Matt }\end{array}$ & Alemania & {$[\mathrm{AS}]$} & {$[\mathrm{TR}]$} & [ODS10] & {$[S E],[S V]$} & {$[\mathrm{CB}]$} \\
\hline$[\mathrm{C} 2]$ & \#SheDrives & Nissan & Automoción & $\begin{array}{l}\text { [a] TBWA/ } \\
\text { RAAD Dubai }\end{array}$ & Arabia Saudí & {$[\mathrm{AS}]$} & {$[\mathrm{TA}]$} & [ODS5] & {$[\mathrm{SE}]$ y $[\mathrm{SV}]$} & {$[C B]$} \\
\hline [C3] & Save our species & Lacoste & Moda & $\begin{array}{l}\text { [a] BETC } \\
\text { Paris }\end{array}$ & Francia & {$[\mathrm{AS}]$} & {$[\mathrm{TC}]$} & [ODS15] & {$[S L]$} & {$[\mathrm{CU}]$} \\
\hline$[\mathrm{C} 4]$ & The Flip & McDonald's & Restauración & $\begin{array}{l}\text { [a] We Are } \\
\text { Unlimited }\end{array}$ & $\begin{array}{l}\text { Estados } \\
\text { Unidos }\end{array}$ & {$[\mathrm{AS}]$} & {$[\mathrm{TA}]$} & [ODS5] & {$[S M],[S V]$} & {$[\mathrm{CU}],[\mathrm{CB}]$} \\
\hline$[\mathrm{P} 1]$ & $\begin{array}{l}\text { Curiosity Cube } \\
\text { Ignites Scientific } \\
\text { Passion }\end{array}$ & $\begin{array}{l}\text { Millipore- } \\
\text { Sigma }\end{array}$ & Industria química & [a] Havas PR & $\begin{array}{l}\text { Estados } \\
\text { Unidos }\end{array}$ & {$[\mathrm{AA}]$} & {$[\mathrm{TB}]$} & [ODS5] & [SE], [SM] & $\begin{array}{c}{[\mathrm{CU}] \mathrm{y}} \\
{[\mathrm{CB}]}\end{array}$ \\
\hline$[\mathrm{P} 2]$ & $\begin{array}{l}\text { Five Years Out/Arrow } \\
\text { Global CSR Program } \\
\text { Annual Report (2017) }\end{array}$ & $\begin{array}{l}\text { Arrow Elec- } \\
\text { tronics }\end{array}$ & $\begin{array}{l}\text { Proveedor de pie- } \\
\text { zas de electrónica }\end{array}$ & [in] & Internacional & [AS] & [TV] & - & {$[\mathrm{SI}]$} & {$[\mathrm{CU}]$} \\
\hline [P3] & \#GiveAToast & $\begin{array}{l}\text { Canadian } \\
\text { Tire }\end{array}$ & $\begin{array}{l}\text { Tiendas de } \\
\text { neumáticos }\end{array}$ & $\begin{array}{l}\text { [a] Commu- } \\
\text { nity }\end{array}$ & Canadá & {$[\mathrm{AB}]$} & [TD] & [ODS10] & {$[\mathrm{SF}]$} & {$[C B]$} \\
\hline [P4] & $\begin{array}{l}\text { Arrow Charitable: } \\
\text { Giving Back Where We } \\
\text { Live and Work }\end{array}$ & $\begin{array}{l}\text { Arrow Elec- } \\
\text { tronics }\end{array}$ & $\begin{array}{l}\text { Proveedor de pie- } \\
\text { zas de electrónica }\end{array}$ & [in] & $\begin{array}{l}\text { Estados Uni- } \\
\text { dos y Canadá }\end{array}$ & $\begin{array}{l}{[\mathrm{AE}] /} \\
{[\mathrm{AS}]}\end{array}$ & {$[\mathrm{TV}]$} & - & {$[\mathrm{SH}]$} & {$[\mathrm{CB}]$} \\
\hline
\end{tabular}




\begin{tabular}{|c|c|c|c|c|c|c|c|c|c|c|}
\hline [P5] & Hurricane Harvey & VSP Global & $\begin{array}{l}\text { Servicios y } \\
\text { productos de } \\
\text { oftalmología }\end{array}$ & - & $\begin{array}{l}\text { Estados } \\
\text { Unidos }\end{array}$ & {$[\mathrm{AC}]$} & [TS] & [ODS3] & {$[\mathrm{SH}],[\mathrm{SV}]$} & {$[\mathrm{CB}]$} \\
\hline [P6] & $\begin{array}{l}\text { Zero Hunger Zero } \\
\text { Waste }\end{array}$ & $\begin{array}{l}\text { The Kroger } \\
\text { Co. }\end{array}$ & Supermercados & [in] & $\begin{array}{l}\text { Estados } \\
\text { Unidos }\end{array}$ & {$[\mathrm{AH}]$} & {$[\mathrm{TH}]$} & [ODS2] & {$[\mathrm{SA}]$} & {$[\mathrm{CU}]$} \\
\hline$[\mathrm{P} 7]$ & Girls4Tech & Mastercard & $\begin{array}{l}\text { Sistema de pago } \\
\text { electrónico }\end{array}$ & [in] & $\begin{array}{l}\text { Estados } \\
\text { Unidos, Reino } \\
\text { Unido, Aus- } \\
\text { tralia, Polonia, } \\
\text { Singapury } \\
\text { China }\end{array}$ & {$[\mathrm{AA}]$} & {$[\mathrm{TB}]$} & [ODS5] & $\begin{array}{c}{[\mathrm{SH}],[\mathrm{SE}]} \\
{[\mathrm{SV}]}\end{array}$ & {$[\mathrm{CB}]$} \\
\hline$[\mathrm{P} 8]$ & $\begin{array}{l}\text { Northrop Grumman } \\
\text { Foundation Teachers } \\
\text { Academy }\end{array}$ & $\begin{array}{l}\text { Northrop } \\
\text { Grumman } \\
\text { Foundation } \\
\text { y National } \\
\text { Science } \\
\text { Teachers } \\
\text { Association }\end{array}$ & $\begin{array}{l}\text { Industria ae- } \\
\text { roespacial y de } \\
\text { defensa }\end{array}$ & [in] & $\begin{array}{l}\text { Estados } \\
\text { Unidos y } \\
\text { Australia }\end{array}$ & {$[\mathrm{AA}]$} & [TB] & [ODS4] & {$[S E]$} & {$[\mathrm{CU}]$} \\
\hline [P9] & Read to Succeed & KPMG LLP & $\begin{array}{l}\text { Servicios de } \\
\text { auditoría, de } \\
\text { asesoramiento } \\
\text { legal y fiscal, y de } \\
\text { asesoramiento } \\
\text { financiero y de } \\
\text { negocio }\end{array}$ & [in] & $\begin{array}{l}\text { Estados } \\
\text { Unidos }\end{array}$ & {$[A B]$} & [TD] & [ODS10] & {$[\mathrm{SE}]$} & {$[\mathrm{CB}]$} \\
\hline [P10] & $\begin{array}{l}\text { Honeywell Humani- } \\
\text { tarian Relief Fund: } \\
\text { Providing Disaster } \\
\text { Relief Directly to } \\
\text { Employees and their } \\
\text { Communities }\end{array}$ & Honeywell & $\begin{array}{l}\text { Producción de } \\
\text { productos de } \\
\text { consumo, servicios } \\
\text { de ingeniería y } \\
\text { sistemas aeroes- } \\
\text { paciales }\end{array}$ & [in] & $\begin{array}{l}\text { Estados Uni- } \\
\text { dos, Puerto } \\
\text { Rico, México y } \\
\text { Rumanía }\end{array}$ & {$[\mathrm{AC}]$} & [TG] & [ODS13] & {$[\mathrm{SF}]$} & {$[\mathrm{CB}]$} \\
\hline [P11] & $\begin{array}{l}\text { Honeywell Educators } \\
\text { at Space Academy }\end{array}$ & Honeywell & $\begin{array}{l}\text { Producción de } \\
\text { productos de } \\
\text { consumo, servicios } \\
\text { de ingeniería y } \\
\text { sistemas aeroes- } \\
\text { paciales }\end{array}$ & [in] & $\begin{array}{l}\text { Estados } \\
\text { Unidos y } 33 \\
\text { países más }\end{array}$ & {$[\mathrm{AA}]$} & {$[\mathrm{TB}]$} & [ODS4] & {$[\mathrm{SE}]$} & {$[\mathrm{CB}]$} \\
\hline [P12] & $\begin{array}{l}\text { SpartanNash } \\
\text { Foundation }\end{array}$ & $\begin{array}{l}\text { Spartan- } \\
\text { Nash }\end{array}$ & $\begin{array}{l}\text { Distribuidor de } \\
\text { alimentos y super- } \\
\text { mercados }\end{array}$ & [in] & $\begin{array}{l}\text { Estados } \\
\text { Unidos }\end{array}$ & {$[\mathrm{AF}]$} & [TV] & [ODS2] & {$[S F]$} & {$[\mathrm{CB}]$} \\
\hline [P13] & Girls4Tech & Mastercard & $\begin{array}{l}\text { Sistema de pago } \\
\text { electrónico }\end{array}$ & [in] & $\begin{array}{l}\text { Estados } \\
\text { Unidos, } \\
\text { Reino Unido, } \\
\text { Australia, } \\
\text { Polonia, Sin- } \\
\text { gapur y China }\end{array}$ & {$[\mathrm{AA}]$} & {$[\mathrm{TB}]$} & [ODS5] & $\begin{array}{c}{[\mathrm{SH}],[\mathrm{SE}]} \\
{[\mathrm{SV}]}\end{array}$ & {$[\mathrm{CB}]$} \\
\hline [P14] & $\begin{array}{l}\text { TeenDrive365 In } \\
\text { School }\end{array}$ & $\begin{array}{l}\text { Toyotay } \\
\text { Discovery } \\
\text { Education }\end{array}$ & $\begin{array}{l}\text { Fabricantes de } \\
\text { automóviles }\end{array}$ & [in] & Internacional & [AA] & {$[\mathrm{TX}]$} & [ODS3] & [SE], [SM] & {$[\mathrm{CB}]$} \\
\hline [P15] & Good Ingredients & $\begin{array}{l}\text { Wawel SA } \\
\text { y Garden } \\
\text { of Words } \\
\text { Group }\end{array}$ & $\begin{array}{l}\text { Fabricante de } \\
\text { dulces }\end{array}$ & [in] & Polonia & {$[\mathrm{AG}]$} & {$[\mathrm{TI}]$} & [ODS12] & [SM] & {$[\mathrm{CU}]$} \\
\hline [P16] & $\begin{array}{l}\text { SpareFoot Diversity } \\
\text { and Inclusion Task } \\
\text { Force }\end{array}$ & SpareFoot & $\begin{array}{l}\text { Almacenamiento } \\
\text { de información } \\
\text { personal }\end{array}$ & [in] & $\begin{array}{l}\text { Estados } \\
\text { Unidos }\end{array}$ & {$[\mathrm{AE}]$} & {$[\mathrm{TA}]$} & [ODS5] & {$[\mathrm{SE}]$} & {$[\mathrm{CU}]$} \\
\hline [P17] & XTEAM & $\begin{array}{l}\text { Berkshire } \\
\text { Bank }\end{array}$ & Banca & [in] & $\begin{array}{l}\text { Estados } \\
\text { Unidos }\end{array}$ & [AS] & [TV] & - & {$[S E]$} & {$[\mathrm{CB}]$} \\
\hline [P18] & Life of a Can & $\begin{array}{l}\text { Novelis and } \\
\text { Discovery } \\
\text { Education }\end{array}$ & $\begin{array}{l}\text { Productor de alu- } \\
\text { minio y reciclaje } \\
\text { de latas }\end{array}$ & [in] & $\begin{array}{l}\text { Estados } \\
\text { Unidos }\end{array}$ & $\begin{array}{c}{[A A] y} \\
A B]\end{array}$ & {$[\mathrm{TC}]$} & [ODS13] & {$[\mathrm{SV}]$} & {$[\mathrm{CB}]$} \\
\hline [P19] & $\begin{array}{l}\text { Organizational } \\
\text { Transparency }\end{array}$ & $\begin{array}{l}\text { Western } \\
\text { Area Power } \\
\text { Administra- } \\
\text { tion }\end{array}$ & $\begin{array}{l}\text { Energía hidroeléc- } \\
\text { trica (Departa- } \\
\text { mento de Energía } \\
\text { de EEUU) }\end{array}$ & [in] & $\begin{array}{l}\text { Estados } \\
\text { Unidos }\end{array}$ & [AS] & [TM] & [ODS16] & [SM], [SV] & {$[\mathrm{CU}]$} \\
\hline [P20] & - & -- & - & $\begin{array}{l}\text { [a] Yulu } \\
\text { Public } \\
\text { Relations }\end{array}$ & $\begin{array}{l}\text { Estados Uni- } \\
\text { dos y Canadá }\end{array}$ & [AO] & - & - & - & - \\
\hline [P21] & $\begin{array}{l}\text { Creating a Healthier } \\
\text { Generation of Ameri- } \\
\text { cans in } 2017\end{array}$ & $\begin{array}{l}\text { Anthem } \\
\text { Foundation }\end{array}$ & $\begin{array}{l}\text { Fundación que } \\
\text { promueve la salud } \\
\text { (empresa de pro- } \\
\text { ductos y servicios } \\
\text { sanitarios) }\end{array}$ & [in] & $\begin{array}{l}\text { Estados } \\
\text { Unidos }\end{array}$ & {$[\mathrm{Al}]$} & [TS] & [ODS3] & $\begin{array}{c}{[\mathrm{SF}],[\mathrm{SE}]} \\
{[\mathrm{SM}]}\end{array}$ & {$[\mathrm{CU}]$} \\
\hline
\end{tabular}


Categoría Premios. Subcategorías:

[I1] IPRA Golden Awards;

[C] Cannes Lions PR;

[P] PR Daily Awards.

Categoría Sujeto promotor: nombre de la empresa o institución que ha financiado la campaña de RSC.

Categoría Sector económico: descripción del sector económico del sujeto promotor.

Categoría Sujeto ejecutor:

[in] departamento de comunicación integrado en la organización;

[a] agencia o consultora de comunicación y Relaciones Públicas.

Categoría Área geográfica: internacional o nombre del país o países.

Categoría Público:

[AA] estudiantes de secundaria;

[AB] niños;

[AS] sociedad en general;

[AE] empleados;

[AC] damnificados por catástrofes naturales;

$[\mathrm{AH}]$ personas que sufren hambre;

[AF] población vulnerable;

[AG] consumidores de dulces;

[AI] enfermos;

[AO] organizaciones que quieran apostar por la RSC.

Categoría Tema RSC:

[TA] igualdad de género y empoderamiento de la mujer:

[TB] promoción vocaciones STEM;

[TC] protección del medio ambiente;

[TD] igualdad de oportunidades;

[TE] lucha contra el acoso escolar;

[TG] ayuda a paliar efectos catástrofes naturales;

[TH] reducir el hambre y el desperdicio de alimentos;

[TI] producción de dulces con productos más naturales;

[TR] lucha contra el racismo;

[TO] reducción de la orfandad;

[TM] transparencia contra la malversación de fondos;

[TS] salud;

[TX] reducir muertes por accidentes de coche;

[TV] múltiples acciones de RSC.

Categoría ODS:

[ODS1] Erradicar la pobreza en todas sus formas en todo el mundo;

[ODS2] Poner fin al hambre, conseguir la seguridad alimentaria y una mejor nutrición, y promover la agricultura sostenible;

[ODS3] Garantizar una vida saludable y promover el bienestar para todos en todas las edades;

[ODS4] Garantizar una educación de calidad inclusiva y equitativa, y promover las oportunidades de aprendizaje permanente para todos

[ODS5] Alcanzar la igualdad entre los géneros y empoderar a todas las mujeres y niñas;

[ODS6] Garantizar la disponibilidad y la gestión sostenible del agua y el saneamiento para todos;

[ODS7] Asegurar el acceso a energías asequibles, fiables, sostenibles y modernas para todos;

[ODS8] Fomentar el crecimiento económico sostenido, inclusivo y sostenible, el empleo pleno y productivo, y el trabajo decente para todos;

[ODS9] Desarrollar infraestructuras resilientes, promover la industrialización inclusiva y sostenible, y fomentar la innovación;

[ODS10] Reducir las desigualdades entre países y dentro de ellos;

[ODS11] Conseguir que las ciudades y los asentamientos humanos sean inclusivos, seguros, resilientes y sostenibles;

[ODS12] Garantizar las pautas de consumo y de producción sostenibles;

[ODS13] Tomar medidas urgentes para combatir el cambio climático y sus efectos;

[ODS14] Conservar y utilizar de forma sostenible los océanos, mares y recursos marinos para lograr el desarrollo sostenible;

[ODS15] Proteger, restaurar y promover la utilización sostenible de los ecosistemas terrestres, gestionar de manera sostenible los bosques, combatir la desertificación y detener y revertir la degradación de la tierra, y frenar la pérdida de diversidad biológica;

[OD16] Promover sociedades pacíficas e inclusivas para el desarrollo sostenible, facilitar acceso a la justicia para todos y crear instituciones eficaces, responsables e inclusivas a todos los niveles;

[ODS17] Fortalecer los medios de ejecución y reavivar la alianza mundial para el desarrollo sostenible.

Categoría Técnicas y tácticas:

[SC] celebrity endorsement;

[SE] organización de eventos;

[SF] fundraising;

[SG] gamification

[SM] relaciones con los medios;

[SI] informes;

[SH] participación de empleados en programas de voluntariado;

[SV] video content

[SA] cause advocacy;

[SS] esponsorización

[SL] lanzamiento de producto de edición limitada con fines benéficos.

Categoría Canales:

[CU] canales unidireccionales;

[CB] canales bidireccionales 